\title{
Das Kulturanthropologische Institut Oldenburger Münsterland
}

Anders als in Nordrhein-Westfalen mit seinen Landschaftsverbänden gibt es in Niedersachsen keine Forschungsstelle, die sich landesweit der Volkskunde bzw. der Alltagskultur widmet. Dass hier Forschungsbedarf besteht, ist jedoch zumindest im Oldenburger Münsterland erkannt worden. Das Oldenburger Münsterland bzw. Südoldenburg, d. h. das Gebiet der Landkreise Cloppenburg und Vechta, ist historisch ein Teil des Niederstifts Münster; zwischen 1815 und 1946 war es Teil des Landes Oldenburg. Gegenwärtig gehören rund 55 Prozent der Einwohner des Landkreises Cloppenburg und rund 6o Prozent der Einwohner des Kreises Vechta der katholischen Kirche an.

Nach geographischen Kriterien wie der Einwohnerdichte, hoher Anteile land- und forstwirtschaftlicher Flächen oder der Entfernung von großen Zentren ist Südoldenburg ein sehr ländliches Gebiet. ${ }^{1}$ Anhaltend stark ist die Stellung der Landwirtschaft. Die Agrarwirtschaft ist durch die Veredelung, besonders durch die Schweine- und Geflügelmast, an Standorten mit ertragreichen Böden auch durch den Obst- und Gemüseanbau zu großer Intensität gelangt. Rund um die Landwirtschaft hat sich eine sehr dichte vor- und nachgelagerte Industrie entwickelt. Die Wirtschaft der Region wächst seit den 196oer Jahren kontinuierlich, und dies wirkt sich nicht zuletzt auf die Bevölkerungszahlen aus: Im Gegensatz zu den meisten anderen ländlichen Gebieten verlieren die Orte des Oldenburger Münsterlandes keine Einwohner, sondern gewinnen neue hinzu. Zur Wirklichkeit der Region gehört freilich auch, dass sie bisweilen zwiespältig wahrgenommen wird; im Vordergrund steht hier vor allem die Intensivtierhaltung.

Hinter den Stereotypen gerät die kulturelle Vielfalt des Südoldenburger Raumes leicht aus dem Blick. Das Kulturanthropologische Institut Oldenburger Münsterland übernimmt die Aufgabe, das alltagskulturelle Wissen über die Region zu erweitern. Es ermöglicht eine institutionell abgesicherte kulturwissenschaftliche Forschung und damit die Möglichkeit, über die Laufzeit eines Projektes hinaus kulturelle Entwicklungen zu verfolgen. Durch seine Forschungs- und Dokumentationsarbeit wird das Institut nicht zuletzt in der Lage sein, für die Zukunft relevante Auskünfte zum Leben und Arbeiten in der Region zu geben. Seine Forschungsergebnisse will das Institut durch Vorträge, Aufsätze, Monographien, Tagungen und Ausstellungen an die Öffentlichkeit bringen.

Die Geschichte des Instituts setzt im Oktober 2018 ein, als die Landkreise Cloppenburg und Vechta gemeinsam mit dem Museumsdorf Cloppenburg und der Universität Vechta das Institut für Kulturanthropologie des Oldenburger Münsterlandes e. V. gründeten. Die für den Institutsbetrieb nötigen Mittel werden durch die Landkreise

$1 \mathrm{Zu}$ den Kriterien des ländlichen Raumes s. zuletzt Werner Bätzing: Das Landleben. Geschichte und Zukunft einer gefährdeten Lebensform. München 2020, S. 16-21, mit weiteren Hinweisen. 
Cloppenburg und Vechta aufgewendet. Bundesweit ist es eine Besonderheit, dass zwei Landkreise ein kulturwissenschaftliches Forschungsinstitut fördern.

Mit der Einstellung der Geschäftsführerin Prof. Dr. Christine Aka zum Mai 2019 nahm das Institut seine Tätigkeit auf. Es ist in unmittelbarer Nachbarschaft des Museumsdorfs Cloppenburg angesiedelt und profitiert auch von seiner Infrastruktur. Der Universität Vechta ist die Einrichtung als An-Institut verbunden.

Von seinem Zuschnitt her gehört das Institut zu den kleineren einschlägigen Landesstellen. Neben der Geschäftsführerin sind ein wissenschaftlicher Mitarbeiter und eine wissenschaftliche Volontärin in der Einrichtung beschäftigt. Im Rahmen des Projektes „Missionarinnen global lokal - Netzwerke im katholischen Milieu“ wurde zum September 2020 ein weiteres Volontariat ausgeschrieben. Als Beispiele für die Arbeit des Instituts seien hier drei aktuell verfolgte Projekte vorgestellt. ${ }^{2}$

\section{Missionarinnen global lokal - Netzwerke im katholischen Milieu (Christine Aka)}

Innerhalb der katholischen Kirche haben durch Missionare und Missionarinnen fundierte Formen global existierender Netzwerkstrukturen eine lange Tradition und sind zumeist positiv besetzt. Sie haben dabei vielfältige, nicht nur innerkirchliche Auswirkungen auf interkulturelle Beziehungen und auf das Wissen über die Welt. Ihre Bedeutung wurde bisher aber weder in der Kulturanthropologie noch in den weiterhin betriebenen und sich neu justierenden Missionswissenschaften untersucht. Der biblische Aussendungsauftrag und das christliche Ideal der Caritas führten im Kontext von Imperialismus, Kolonialismus und der Entstehung des katholischen Milieus vor allem im späten 19. und frühen 20. Jahrhundert zu einer extremen Begeisterung für den Missionarsberuf.

In den Niederlanden wurden in den 1980er bis 20ooer Jahren fast 7000 Missionare und Missionarinnen interviewt. In Deutschland wurde dergleichen versäumt. Die Rekonstruktion der bis heute eng verknüpften Netzwerke kann somit nur noch durch eine kulturwissenschaftliche Kombination vieler verstreuter Quellen erfolgen. Sie erfordert einen guten Zugang zum Feld in katholischen Lebenswelten. ${ }^{3}$

Das hier vorgestellte Projekt fragt konkret nach Mustern von „Beziehungsarbeit“ zwischen Akteuren in der "Mission“ und ihrer Heimat. Ein Schwerpunkt liegt auf den etwa 350 zwischen 1900 und 1970 aus dem Oldenburger Münsterland ausgereisten Missionarinnen, deren Namen bisher durch die Auswertung von Ortschroniken gefunden werden konnten. Im Gegensatz zum männlichen Klerus wurden sie niemals systematisch erfasst. Anhand schriftlicher Quellen, vor allem Briefwechseln, aber auch Reise- und Tätigkeitsberichten in der lokalen Presse und, soweit noch möglich, durch

2 Weitere Informationen zum Institut gibt die Internetseite kai-om.de.

3 Christine Egger: Transnationale Biographien. Die Missionsbenediktiner von St. Ottilien in Tanganjika 1922-1965. Köln, Weimar, Wien 2016. 
Interviews, soll untersucht werden, welches Wissen über die fremde Welt vermittelt und auch interpretiert wurde, welche Bedeutung gerade Missionarinnen im Bereich der "Carework“ und damit in einem weiten Bereich der „Entwicklungshilfe“ spielten und wie sie ihr Leben immer auch in den Dienst des Spendensammeln stellten.

Männliche Missionare, zumeist in priesterlicher Funktion, sollen in den Fällen berücksichtigt werden, in denen sie z.B. eine Rolle beim Phänomen der sogenannten Kettenberufungen spielten. Auch waren es meistens Männer, die Kontakte zu Großspendern nutzten, um Projekte zu realisieren, in denen die Nonnen zumeist die realen karitativen oder lehrenden Tätigkeiten übernahmen.

Missionsschulen waren nicht selten der Ausgangspunkt für die Herausbildung lokaler Eliten. Die dort Ausgebildeten wurden in die sich vergrößernden Netzwerke eingebunden. Schon in den 1920er Jahren entwickelte sich der Aufbau der Berufs- und Erwachsenenbildung zum Schwerpunkt missionarischen Schulwesens und damit zu einer Grundlage für den Beginn eines Prozesses, der sich von der reinen Bekehrungsund Zivilisierungsabsicht löste und Vorformen christlicher Entwicklungszusammenarbeit hervorbrachte, die bis heute fortbestehen. Die Mädchenausbildung und auch die Krankenpflege lagen dabei gänzlich in den Händen der Missionarinnen.

Interlokale globale Kommunikationsbeziehungen und implementierte Wissensvermittlung über das je andere folgen somit noch heute den seit damals gewachsenen und wenig beachteten Strukturen. Noch heute werden Container gepackt, reisen Jugendgruppen nach Afrika, gehen Großnichten und -neffen als Missionare auf Zeit zu den Wirkungsstätten ihrer Verwandten. Ob Malawi-Kreis, Indien-Hilfe, Togo-Verein oder Brasilienunterstützerclub - in christlichen Kirchengemeinden findet man noch immer viele Aktive, die Projekte in der sogenannten Mission, z. B. die Tätigkeit einer heute meist hochbetagten Nonne aus der Heimatgemeinde, finanzieren.

Spuren der vielen Tanten, Cousinen, Geschwister oder Nachbarinnen reichen in die ganze Welt, von Südamerika und Afrika nach Japan und in den pazifischen Raum, aber auch in die USA, nach Kanada, nach Dänemark oder Island.

MissionarInnen waren niemals isoliert; sie standen in Austauschbeziehungen zu ihrem Orden, zu ihren Heimatgemeinden und zu ihren Unterstützern. Ansätze, Ideen und Handlungspraktiken wurden somit transnational und trotz regionaler Schwerpunkte global verhandelt, auch wenn die Kommunikation zäh und langwierig war, über Fischerboote zu Dampfschiffen, über Eisenbahnen und Postkutschen. Angesichts der Hindernisse, die überwunden wurden, nimmt es nicht wunder, dass heute tätige Missionarinnen noch mit über achtzig Jahren beispielsweise die Handhabung von Skype und WhatsApp beherrschen.

Ein Resultat der skalierenden Vernetzungen ist nicht zuletzt auch darin zu sehen, dass heute aus den erst vor wenigen Generationen christianisierten Weltregionen der geistliche Nachwuchs rekrutiert wird, der die katholische Kirche als Dienstleistungsbetrieb auch im westlichen Europa weiter funktionieren lässt. Pfarrer aus Indien oder aus Nigeria unterstützen heute ihre Kollegen auch im Oldenburger Münsterland und 
knüpfen dabei ganz selbstverständlich neue Unterstützernetze für Projekte in ihrer Heimat. Auch dies wird in dem Projekt im Blick behalten.

\section{Strukturwandel und bäuerliches Selbstverständnis - Landwirtschaft im Oldenburger Münsterland (Thomas Schürmann)}

Das Oldenburger Münsterland gehört zu den intensivsten Agrarregionen Europas. Einen ersten Hinweis hierauf geben die Schlachtzahlen: Rund 20 Prozent der in der Bundesrepublik Deutschland geschlachteten Schweine, 12 Prozent der Hähnchen und 15 Prozent der Puten verließen 2019 Schlachthöfe in den Landkreisen Cloppenburg und Vechta. Die Veredelungswirtschaft ermöglichte es, dass in einer Region mit meist ertragsarmen Böden eine hochproduktive Landwirtschaft entstand und sich um sie ein stark verdichtetes vor- und nachgelagertes Gewerbe entwickelte.

Seit Jahrzehnten steht die Landwirtschaft jedoch unter wachsendem Druck. Aufgrund strengerer Umweltauflagen wird es schwieriger, den durch die Tierhaltung anfallenden Wirtschaftsdünger auszubringen. In der Gesellschaft sehen sich die Landwirte einem wachsenden Unverständnis ausgesetzt. Auch der finanzielle Druck auf die Betriebe erhöht sich: Die Pachtzinsen für landwirtschaftliche Flächen haben mit bis um 1500 Euro pro Hektar Höhen erreicht, die viele Betriebe an die Grenzen ihrer Leistungsfähigkeit bringen. Die meisten Höfe sind jedoch mit geringen Flächen ausgestattet und darauf angewiesen, Land hinzuzupachten.

Die Zukunft vieler Höfe ist ungewiss. Innerhalb der Bundesrepublik gilt die Hofnachfolge bei nur 30 Prozent der landwirtschaftlichen Betriebe als gesichert. Auch in Südoldenburg stehen viele Höfe spätestens mit dem Generationenwechsel vor der Betriebseinstellung.

Das Projekt untersucht auf der Grundlage erzählender Interviews, wie sich die Betroffenen, d.h. die Landwirte und Landwirtinnen, in dieser Situation verhalten, wie sie ihre Aussichten einschätzen und welche Strategien sie entwickeln. Auch das Verhältnis zur Tradition ist von Bedeutung: Welche Rolle spielt das Hofdenken, nach dem die persönlichen Belange weitgehend dem Weiterbestehen des Betriebes untergeordnet werden, und welche Rolle spielt der Gedanke der Freiheit des selbständigen Landwirts? Sind sie eine mentale Stütze, oder sind sie eher ein Ansporn zur Selbstausbeutung? Werden hergebrachte Formen des Erbrechts, die den Hofnachfolger deutlich privilegieren, weiterhin weitgehend fraglos hingenommen? Wie haben sich der Alltag und das Berufsbild der Landwirte verändert? Über die Ebene der Betriebe hinaus fragt das Projekt, wie Dörfer sich entwickeln, in denen oft nur noch wenige landwirtschaftliche Betriebe aktiv sind, und welche Rolle die Landwirte, die jahrhundertelang die tonangebende Bevölkerungsgruppe waren, in den Gemeinden spielen. Diese Fragen sind über den Agrarsektor hinaus von Bedeutung, denn mit der Landwirtschaft ändert sich der Charakter des gesamten ländlichen Raumes. 


\section{Abtanzball, Einmehlen und Schachtelkranz - Jugendkultur im Oldenburger Münsterland \\ (Malaika Winzheim)}

Begünstigt durch eine der höchsten Geburtenraten ist das Oldenburger Münsterland eine der jüngsten Regionen in Deutschland. Ein breites Angebot an kulturellen Angeboten und eine blühende Vereinsdichte tragen ebenfalls dazu bei, dass sich junge Menschen hier wohlfühlen. Dieser Umstand und die Tatsache, dass sich hier eine der wirtschaftlich stärksten Regionen der Bundesrepublik befindet, erklärt den enormen Anstieg der Einwohnerzahlen der letzten dreißig Jahre. ${ }^{4}$ Grund genug für das Institut, die regionale Jugendkultur mit ihren Ausprägungen und die gesellschaftlichen und vor allem die nachbarschaftlichen Strukturen genauer ins Visier zu nehmen. Im Rahmen des wissenschaftlichen Volontariats sind eine Sonderausstellung, die im $\mathrm{Mu}$ seumsdorf Cloppenburg zu sehen sein wird, und eine Publikation geplant, welche einen Ausschnitt der regionaltypischen Jugendkultur zeigen werden.

Grundlage der Forschung bilden Interviews mit jungen Menschen aus beiden Landkreisen. Auch private Fotografien werden hinzugezogen. Geplant waren zunächst auch teilnehmende Beobachtungen, bei denen eigenes Bildmaterial generiert werden sollte, jedoch machten die Corona-Pandemie und die damit einhergehenden Einschränkungen dieses Vorhaben bis jetzt nur sehr eingeschränkt möglich. Auswirkungen hatte dies auch auf die Interviewführung: Ein Teil der Interviews wurde über Skype geführt. Dies war jedoch kein Hindernis, weder für die Forscherin noch für die Interviewten, die mit großer Begeisterung von ihrem Leben als Jugendliche und junge Erwachsene in der Region erzählen. Durch diese akteurszentrierte Herangehensweise wird ein möglichst aktuelles und umfangreiches Bild der Alltagswelten junger Menschen im Oldenburger Münsterland erfasst. Das Projekt untersucht die Kennzeichen der dortigen Jugendkultur und berücksichtigt dabei besonders die Funktionen von Cliquen, Vereinen, Nachbarschaften und anderen sozialen Verbänden.

Die Interviews beschränken sich auf Personen im Alter von 14 bis 30 Jahren. Dies ergibt sich aus folgenden Faktoren: Mit 14 Jahren begehen viele Jugendliche im Anschluss an den Besuch einer Tanzschule einen Abtanzball. Für die meisten von ihnen bedeutet das, ihre erste richtige Party zu feiern - freilich unter elterlicher Aufsicht. Deshalb markiert dieses einschneidende Erlebnis den Beginn des Forschungsprojekts. Zum 16. Geburtstag findet in Teilen Südoldenburgs ein außergewöhnlicher, aber charakteristischer Brauch statt: Die Jugendlichen werden von ihren Freunden „eingemehlt“. Mit diesem Ritual wird der Übergang in eine Lebensphase mit neuen Freiheiten gefeiert. Neben der Wertschätzung, die sich in selbstverfassten Gedichten und Sprüchen unterhaltsamer Natur äußert, die auf Schildern für die Nachbarschaft gut sichtbar am Haus angebracht werden, spielt selbstverständlich auch der Spaßfaktor des Vorgangs eine nicht zu unterschätzende Rolle.

4 Daten in https://www.oldenburger-muensterland.de/service/daten-region-om/? (8.7.2020). 
Zum 25. Geburtstag ist es üblich, den unverheirateten Frauen einen Schachtelkranz vorbeizubringen. Das Symbol spricht für sich: Sie ist nun offiziell eine alte Schachtel! Den unverheirateten Männern wird zu diesem Ereignis ein Flaschenkranz gebracht; im Schaltjahr wird getauscht. Eigentlich haben wir es hier mit einem Rügebrauch zu tun, wenngleich es heute nicht mehr als solcher empfunden wird, sondern vor allem dazu dient, die bestehenden Freundes- und Bekanntenkreise zu pflegen. ${ }^{5}$

Die obere Altersgrenze des Jugendkultur-Projekts wurde auf 30 Jahre festgelegt, denn durch die Verlängerung der Lebensphase der Adoleszenz fallen noch weitere interessante Bräuche mit in diesen Bereich. Ein recht bekanntes Beispiel dazu ist das Treppenfegen der unverheirateten Männer am 30. Geburtstag. ${ }^{6}$ Unverheiratete Frauen müssen an ihrem 30. Geburtstag Klinken putzen; auch hier wird im Schaltjahr wieder getauscht. Verschiedene Bräuche im Kontext von Ehe und Familiengründung sollen ebenfalls untersucht werden, wie z. B. die Tradition, jungen Eltern anlässlich der Geburt ihres Kindes einen Kilmerstuten ${ }^{7}$ mitzubringen, oder auch der deutlich jüngere Brauch, auf dem Junggesellenabschied den BH der Braut und die Hose des Bräutigams zu verbrennen. Bei der Forschung muss jedoch beachtet werden, dass es nicht „die“ Jugend als homogene Gruppe gibt. Auch die Veränderungen von Bräuchen, etwa ihre Modernisierung oder Individualisierung, finden hier Beachtung. Ein weiterer entscheidender Aspekt des Projekts: Die Bedeutung des Oldenburger Münsterlandes als Heimat für die jungen Menschen wird herausgearbeitet, denn regional gepflegte Bräuche sind immer auch ein Ausdruck lokaler Identitätsstiftung, für die es gerade angesichts der allgegenwärtigen Globalisierung ein verstärktes Bedürfnis zu geben scheint.

Christine Aka, Thomas Schürmann und Malaika Winzheim, Cloppenburg https://doi.org/10.31244/rwz/2020/10

5 Vgl. auch Michael Simon: Bräuche und ihre Zukunft. In: Rheinischer Verein für Denkmalpflege und Landschaftsschutz (Hg.): Fest - Brauch - Event. Regionale Kultur zwischen Tradition und Moderne. Köln 2013, S. 87-104, hier S. 93.

6 Michael Simon: Moderne Brauchinnovation. Geschichte und Funktion des Treppenfegens beim 3o. Geburtstag. In: Jahrbuch für Volkskunde, 21 (1998), S. 157-177; Kerstin EHLERT: Dreißig - ledig - lustig? Moderne Bräuche am 30. Geburtstag (Beiträge zur Volkskunde in Niedersachsen/ Schriftenreihe der Volkskundlichen Kommission für Niedersachsen e. V., 19). Göttingen 2005.

7 Abgeleitet von Kindelbier (Tauffeier). 